\title{
Focusing design and staging design conversations in complex settings through "design documentary" filmmaking
}

Øystein Veland

Departement of Product Design

Norwegian University of Technology and Science (NTNU) oysteinv@hrp.no

\author{
Gisle Andresen \\ Departement of Product Design \\ Norwegian University of Technology and Science (NTNU) \\ gislea@hrp.no
}

\begin{abstract}
This paper explores the use of documentary-style video to support design ethnography and early phase design focusing in a complex industrial context. Based on a design case involving a new interdisciplinary planning tool for an offshore platform operation, we suggest a novel form of "design documentary" filmmaking approach. This work suggests how technically and socially complex design settings may benefit from the use of video as a medium to facilitate and connect different phases and levels of design conversations.
\end{abstract}

User studies, design ethnography, documentary filmmaking, industrial context, collaboration

\section{INTRODUCTION}

\subsection{Overview}

The work presented here is part of a broader effort to explore new interaction design issues that are emerging from an on-going paradigm shift we see taking place in certain advanced industrial settings. Specifically, our research is focused on the design of visualization and interaction solutions that may enable interdisciplinary collaboration in such complex domains. To explore this novel research area in an industrial context we have adopted a research through design approach (Zimmerman et al., 2007) where we pursue new knowledge through engaging with real-world design problems.

This paper is focused on design method rather than product features and describes the use of documentary-style video to support design ethnography, early phase design focusing and conversations with users. This was explored in an industrial design case, and this paper is structured according to the unfolding of events and reflection on issues as they appeared in this design case.

We seek to extract general insights from this case by focusing on generic aspects of its context and reusable elements of the design process we devised in response to it. The significance of our approach and how it relates to other video-based design research is then discussed, before outlining directions for further work.

\subsection{Industrial context}

There is a clear recognition in advanced industrial domains that work processes are being profoundly transformed by the introduction of new communication and collaboration technologies. In the offshore petroleum industry this new technology-enabled, high-efficiency operation paradigm is referred to as Integrated Operations. By leveraging video conferencing and other collaboration tools, high-speed data transfer, information integration and visualization tools, the industry aims to minimize the traditional barriers to efficient operation caused by geographical distances, different systems, and disciplinary and organizational boundaries.

Digital tools capable of connecting workers more closely to their work and to each other are central in this vision of smarter work processes at the individual, team and organizational levels. Still, the high-level visions drawn up by management and technology suppliers have mostly assumed that such tools already exist or will rapidly evolve. And the first generation of such tools are indeed beginning to emerge. They integrate data from different systems and have presentation and interaction capabilities that are definitively more powerful and flexible than those of past systems. But neither the design of these tools themselves nor the processes by which they are designed seem to have undergone any major transformation. Traditional single-user concepts and discipline- 
specific visualization practices remain dominant. Ignoring its potentially major implications from an interface design and end user perspective, these first-generation Integrated Operations tools seem to not fully reflect the radical new ambitions they should serve.

\subsection{The role of interaction design}

Rather than assuming that the basic solutions and design processes of the past are still relevant, our research is based on the position that Integrated Operations may have far-reaching implications both for design products and processes. Interaction design offers a wide range of strategies for background studies, idea development, prototyping, user participation and evaluation. Importantly, it offers concepts and strategies for dealing meaningfully with the early-phase question of "what to build" (Ylirisku et al., 2009).

In the petroleum industry, classic software engineering and systems thinking are still dominant in developing the kind of tools we discuss here. Their evolution is primarily technology-driven and guided by management and IT priorities such as standardization, predictable development and ease of deployment. There is limited use of user studies, participatory design or other approaches that emphasize the end-user and situated perspectives. Thus, interaction design and design thinking seems to be underutilized in this industrial setting. Lack of interest or knowledge of the capabilities of interaction design may partly explain this situation. But it may also be due to interaction designers and their approaches not being sufficiently attuned to the special challenges of engaging effectively with such complex work domains.

In this paper we suggest strategies for approaching a technically and socially complex work domain in ways that are aligned with modern design thinking. We do so based on a particular design case within the wider Integrated Operations realm outlined earlier. In particular, we address how to approach a complex problem domain in the early phases: Exploration of the design context, focusing the design problem, and creating favourable conditions for design collaboration and user involvement.

\section{DESIGN CASE: "INTEGRATED PLANNING"}

The design case that forms the basis for this paper was a project called Integrated Planning Surfaces (IPS) where we sought to design new tools for the planning process of a large offshore petroleum installation. This was chosen as a case because it featured both interdisciplinary collaboration and visualization design as central ingredients. It is beyond the scope of this paper to attempt any comprehensive description of the offshore planning domain. A recent ethnographic study of the collaborative, distributed work processes in this type of planning environment may be found in (Bayerl and Lauche, 2010). However, it is relevant here to convey a sense of the complexity of this domain, because this confronts a designer with significant challenges to which the approach we present here is a response.

Planning offshore operations is a highly complex task that involves careful coordination and scheduling of a large number of activities. Many different disciplines and subcontractors are part of this vast, dynamic "puzzle". Within each individual discipline like drilling, operation, maintenance or modifications, there are complex and often competing priorities and constraints that must be taken into account in the job planning.

As our special focus area within the overall planning process we chose personnel onboard. Limitations on offshore accommodation and helicopter flights are major planning constraints on many installations, and beds and seats are scarce resources that must be skilfully administered because they are so deeply interconnected with everything plan-related. Supervisory planning involves continuous balancing of priorities between disciplines, ensuring that long-term plans are realized, and dealing with the many unforeseen issues that appear. Furthermore, re-planning takes place continuously due to bad weather, illness, operational problems, or cascading effects of reprioritization in other disciplines. Such unforeseen events and dependencies between activities all combine to make offshore planning a highly complex and dynamic process. It inevitably involves many stakeholders, both onshore and offshore: a) Job planners within each discipline, who knows each job and its requirements, b) Booking assistants who finalize orders and manage contact with vendors, c) The planning supervisor who oversees the coordination across disciplines and resolving conflicts, and finally d) Workers whose work periods offshore are being planned.

In the IPS project we sought to establish a deep and holistic understanding of the cognitive and social dimensions of the personnel onboard handling. Our goal was to find opportunities for new collaboration-oriented visualization tools that better integrate the personnel onboard issue with the whole interdisciplinary, multi-location planning process. Well-designed tools could help make this process quicker, more flexible and better informed. 


\section{DESIGNING A DESIGN PROCESS}

In addition to the obvious task of designing the product itself, interaction designers must also design an appropriate design process in response to the particular design context. A "thoughtful interaction design" approach is sensitive to the characteristics of the anticipated product and of the overall context when designing a design process (Löwgren and Stolterman, 2004). Designers must be able to draw on a repertoire of well-proven techniques as well as to invent new methods when needed. We now turn our attention to some key characteristics of the design case and consider some of the opportunities and limitations in using established approaches.

First of all, collaboration and multiple user perspectives were central elements in the design case. CSCW research typically has a rather descriptive approach to collaborative processes and technologies. But from a designer perspective it seems to offer little actual guidance on engaging in design processes involving multi-user and collaborative systems. Members of the design team were also from different disciplinary backgrounds (psychology, engineering, linguistics, and sociology) and were located in three different cities. We therefore sought a design approach that could facilitate also our internal collaboration.

Secondly, the work processes we sought to intervene with involve complex activities taking place at multiple locations, situated in a particular culture. Formal procedures and tools as well as socially constructed practices and locally tailored tools were used to meet the work demands. These are dimensions of the design case that call for a design approach with ethnographical sensitivities. Contextual Design (Beyer and Holtzblatt, 1998) is a popular framework for ethnographically informed design. But it requires relatively extensive data collection and access to users. This kind of intrusion and use of time was fundamentally problematic in the busy operational environment of our case. Our users were key personnel critical to the daily operation of the installation, and it was desirable to interact with them only via brief meetings. Another feature of Contextual Design we found less applicable to our case is the predefined, general-purpose models it uses to speed up the interpretation work. In our design case we wanted a more grounded approach in order to capture any novel issues we expect might emerge from this special interdisciplinary design problem.

A third notable dimension of the design case was how it fundamentally involved mediated work in a technically complex domain. The ultimate purposes, effects and constraints that underlies the work processes originate from an external work domain. Work is mediated via digital artefacts that serve as representations of this external domain. This dimension is central in the Cognitive Work Analysis framework (Vicente, 1999), which captures information requirements by explicit analysis of the work domain. The actual domain knowledge elicitation process is less emphasized here. For a designer entering a highly specialized work domain, the vast number of interrelated actors, objects, and constraints that constitute this design context is highly demanding and potentially overwhelming. A second issue is the lack of a welldefined problem: While problems involving only technical complexity may be amenable to analytic approaches, most real-world problems involve significant social complexity and wickedness which are strong fragmenting forces in a project (Conklin, 2005). A dialogue-oriented approach is recommended for engaging effectively with such problems (Rittel and Webber, 1973). These were helpful concepts in guiding us through the methodological dilemmas we faced at the beginning of this project. It was evident that all these forces of fragmentation are present in our design case. So while the technical complexity might suggest a technical analysis, this was unviable due to the presence of significant social complexity and wickedness, as well as our limited access to workers and their environment. We came to realize that a special design approach had to be devised that incorporated several of the sensitivities and concerns discussed above, while facilitating a process that was fundamentally dialogue-oriented.

A video-based approach seemed the most promising response to the challenges and opportunities we were facing. In their broad review of video-based techniques in design, (Ylirisku and Buur, 2007) show that video can play a broad variety of roles in design activities: From studying what people do, to making sense and editing videos, envisioning the future and finally in provoking change in organizations. Digital camcorders, editing and distribution technologies have also evolved to a point where video is now a cheap, easily available and mouldable medium. Video can meaningfully be perceived as data, as intermediate artefacts during design, and as means of persuasion. Shaping video artefacts involves a series of explicit and implicit decisions on what is filmed, how it is filmed and how it is edited. It is therefore far more than just a medium for capturing objective reality. Another benefit of working with video is that it is a fundamentally social medium that easily creates engagement and involvement. Video may therefore serve both as designer clay and as social glue for those involved in design. 
The use of video is well established in ethnographic fieldwork. (Sperschneider and Bagger, 2003) discuss the challenges of engaging in pure ethnography within the constraints of an industrial setting. They propose more cost-efficient and design-relevant strategies for using video that move beyond pure observation towards engaging users and designers in a more collaborative exploration.

In a similar effort to go beyond using video as a mere note-taking tool in user research, special design documentary films have been proposed as sources of information and inspiration (Raijmakers et al., 2006). By using techniques from documentary film it is possible to portray a design context in ways that preserve ambiguities and paradoxes - not drawing firm conclusions, but opening possibilities for exploration later in the design process. Design work and documentary filmmaking share an inescapable "dialectic between opinions and the 'unadulterated truth'":

\begin{abstract}
"It is impossible for filmmakers to avoid making choices about what is important. At the same time, filmmakers' biases are constrained by the material they have to film: documentaries cannot simply invent the material they use."

"Design teams may thoroughly research the people and situations for which they are designing, but they must also develop a perspective - a prioritized view - to direct their work".
\end{abstract}

The authors argue that neutral observation is often less productive for design purposes, and instead encourage intervention (co-operating with people in the film), and compilation (reusing existing material to construct new narratives, even "radical scavenging" that deeply transforms meanings).

\section{FILMMAKING PROCESS}

For the Integrated Planning Surfaces (IPS) design case we adopted a filmmaking approach inspired by design documentaries. Our film was created through six distinct phases that are described here.

\section{Phase 1: Planning}

From early project meetings we acquired a sufficient overview of the work processes to enable us to make interview appointments with relevant personnel. In a busy operational work environment it was critical to be flexible and sensitive to the circumstances at the time of our visits. A certain amount of planning was still useful to get the most out of each site visit and to obtain the content and quality of material we required. Introductory books on documentary filmmaking and storytelling strategies were also valuable here (Bernard, 2007).

\section{Phase 2: Filming}

In this phase we gathered all the required video material, about 5-6 hours in total. Two members of the design team did the filming and interviewing during three visits to the onshore planning site. Our technical equipment was kept at a minimum to allow rapid and flexible setup and to encourage a sense of informal conversation. A lightweight HD camcorder on tripod without external microphone or additional lighting proved sufficient for our purpose. Individual interviews were done in a separate meeting room, but we also captured valuable footage of the main work locations and their ambient sounds. In a few cases we filmed users at their workplace where they could show examples and demonstrate their tools in context.

\section{Phase 3: Commenting}

The interview footage was distributed to the design team using a video review tool in which each member could insert his or her comments to the material. This helped us familiarize with the material. It also encouraged us to interpret and reflect on the interviews from our separate perspectives, thus postponing the potentially complex task of integrating these diverse perspectives. It later turned out that the nature of these comments were quite consistent within the team. The different comments were joined in the video editing software for use in the next phase.

\section{Phase 4: Filmmaking workshop within the team}

The design team gathered for a one-day collocated session where we analyzed and consolidated our interpretations and outlined the final film's main themes and style.

The first half-day was spent sharing and comparing our comments, sometimes reviewing clips of particular interest together. We searched for emerging patterns in the material based on comments and shared observations. Key issues in the material were thus identified and interesting clips labelled. We also made notes of quotes we wanted to highlight and information that would be effectively conveyed by photos or other visuals.

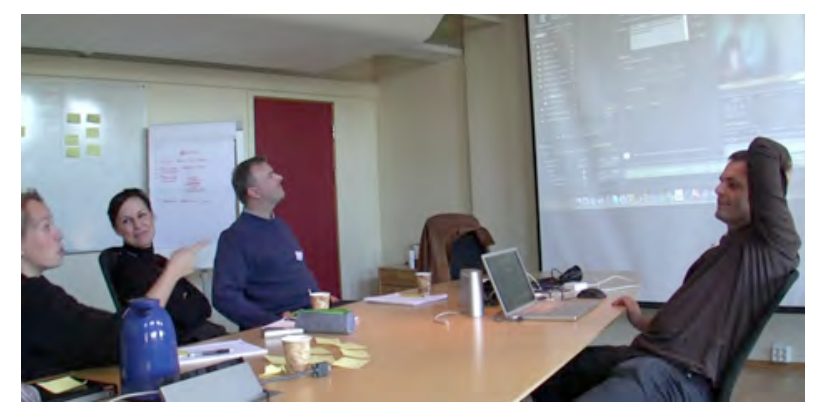

Figure 1: The design team analysing interview material 
Sticky-notes were used as a quick and informal format that could easily be reconfigured. To simplify the video editing after the workshop, we added reference numbers linking the physical sticky notes to digital markers in the video editing software.

In the second half we made decisions on what material to include in the final film and how to structure it, thus developing our "prioritized view". We constructed a storyboard outline on large board where we added sticky-notes with references to clips and ideas for texts and illustrations.

\section{Phase 5: Film completion}

One team member was responsible for the detailed video editing, while the other members were involved via reviewing a series of draft versions. The storyboard created in the previous phase was a starting point for editing the raw interview footage and other material into a 13 minutes long film. This phase took about three weeks and involved creating a detailed structure and composition.

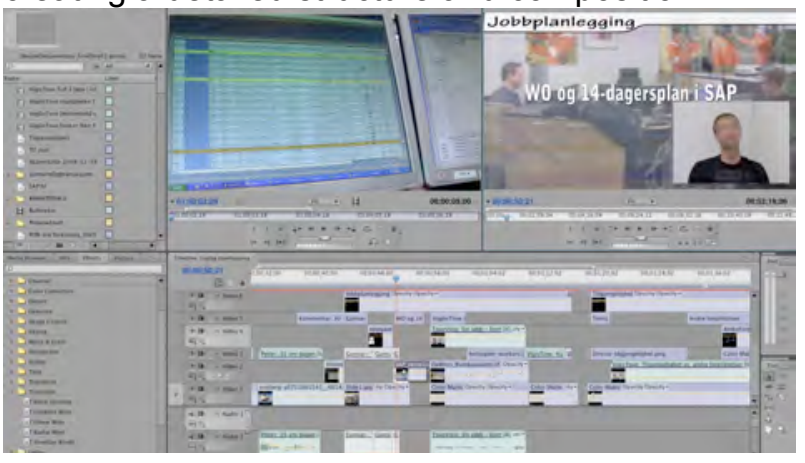

Figure 2: The final film was composed in a video editor by trimming and combining clips, adding text and effects.

The final film employs several techniques to evoke an effective picture of this complex, multifaceted reality and issues for design: Text is used to label topics, while key quotes and metaphors are highlighted using text or illustrative photos. The layout suggests a designer perspective that transcends individual user perspectives, and footage of users and their tools is superimposed on stock photos of the external reality they work with. Several visuals are also created to convey complex information or even suggest design ideas.

\section{Phase 6: Screening session with users}

The final film was shown to a group of users who also had participated in the film. They could then validate or comment on the relevance and correctness of what was presented in the film. We also used the film as a starting point for further conversation to elicit information relevant for our design activities. The context and purpose of the film was explained before it was shown. Afterwards there was time for about a half-hour discussion, which proved to be effectively fuelled by the film.

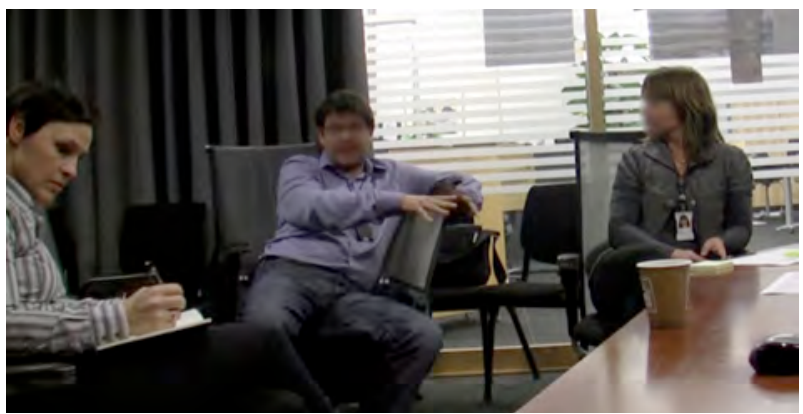

Figure 3: The one-hour screening session included three of the film's protagonists and two design team members.

The general feedback was that we had managed to present a compact, yet fairly correct picture of the challenges in handling the personnel onboard issue. We had also identified further directions for design work that seemed promising.

\section{DISCUSSION}

Creating the kind of film we describe here is both an intellectual and a social process. In participatory design, staging is about creating arenas for conversation and providing constraints that help people gain a similar orientation in their thinking (Ylirisku and Buur, 2007). The material of our filmmaking provides such thinking constraints, while arenas for conversations are created through a series of work situations (commenting footage, editing the final film) and social events (interview sessions, internal workshop, screening session). These multiple, interconnected conversations effectively facilitate focusing - "the iterative process of developing a comprehensive conception of a design object" (Ylirisku et al., 2009). The film artefact is able to absorb our emerging understanding of the empirical reality we studied and carry it between phases and conversations, eventually leading to a basis for new designs.

The idea of video as "social glue" in design may also be related to so-called boundary objects: Serving as facilitators between disparate social worlds within and across organizations, boundary objects are "...objects which both inhabit several intersecting social worlds and satisfy the informational requirements of each of them" (Star and Griesemer, 1989). This concept suggests how we may think of a film artefact as having "boundary object affordances" that make it able to serve multiple stakeholder perspectives at once. For example, while radical scavenging of our material might be inspiring for us as designers, we chose to avoid it to ensure that the film remained meaningful also to those we had interviewed. Careful editing also ensured that all participants appeared as competent and solution-oriented. Some of the visual materials created for the film had a special significance for designers, as early sketch work. 
Common ground theory addresses the role of mutually acknowledged, shared knowledge in communication (Clark, 1996). Early-phase design work in a specialized domain faces special challenges due to the very limited initial common ground between designers and users. After screening the final film, we observed that the conversation proceeded into more detailed and higher-order issues. From a common ground perspective, we may speculate that that the film had rendered visible to the users the substantial knowledge of their world that the designerfilmmakers had acquired. The act of showing the film thus opened access to new, important layers of information for use in the design process.

The term "design documentary" was originally coined in a personas-oriented context. Our work resituates it in an industrial work domain and develops new techniques for this context. This suggests design documentaries as a wider genre, defined by its use of techniques from documentary film and design to combine various materials from the users' empirical reality with designer interpretations to capture a complex design context. Primarily made by designers to form a prioritized view of a complex design context, it also invites users to verify or correct designers' understanding of their domain. Such films may borrow sensitivities from a range of theories and frameworks in design. They should also carefully consider how their content and form connects with different stakeholders in the overall design process.

\section{CONCLUSIONS AND FURTHER WORK}

In this paper we have argued that user-centred and participatory approaches are underutilized in industrial design settings characterized by high technical and social complexity. At the same time, high-performance organizations call for powerful digital tools and would benefit from effective use of interaction design thinking. Our work shows that documentary-style filmmaking can help stage efficient design conversations in this type of context and facilitate the early-phase focusing. We also suggest strategies for making and using such films effectively.

To provide makers of design documentaries with an effective repertoire, a broader range of reusable film techniques should be published based on case studies from different contexts.

A deeper theoretical understanding of video in design conversations may be gained through further use of concepts from CSCW research, such as boundary objects and common ground theory.
Acknowledgements: This research was sponsored by the Center for Integrated Operations in the Petroleum Industry, and was performed in collaboration with Harald Sleire, Kristin Halvorsen and Aud-Marit Wahl from SINTEF Marintek. We also thank the personnel who participated in the IPS project for their invaluable contributions.

\section{REFERENCES}

Bayerl, P. \& Lauche, K. (2010) Technology Effects in Distributed Team Coordination - HighInterdependency Tasks in Offshore Oil Production. Computer Supported Cooperative Work (CSCW), 19, 1-35.

Bernard, S. C. (2007) Documentary Storytelling, Elsevier

Beyer, H. \& Holtzblatt, K. (1998) Contextual design: defining customer-centered systems, Morgan Kaufmann Pub

Clark, H. H. (1996) Using language, Cambridge University Press Cambridge.

Conklin, J. (2005) Dialogue mapping: building shared understanding of wicked problems, John Wiley \& Sons New York.

Löwgren, J. \& Stolterman, E. (2004) Thoughtful interaction design, MIT Press

Raijmakers, B., Gaver, W. W. \& Bishay, J. (2006) Design documentaries: inspiring design research through documentary film. In Proceedings of the 6th conference on Designing Interactive systems, University Park, PA, 229-238, ACM

Rittel, H. W. J. \& Webber, M. M. (1973) Dilemmas in a general theory of planning. Policy Sciences, 4, 155-169.

Sperschneider, W. \& Bagger, K. (2003) Ethnographic fieldwork under industrial constraints: Toward design-in-context. International Journal of Human-Computer Interaction, 15, 41-50.

Star, S. L. \& Griesemer, J. R. (1989) Institutional Ecology, 'Translations' and Boundary Objects: Amateurs and Professionals in Berkeley's Museum of Vertebrate Zoology, 1907-37. Studies of Social Science, 19, 387-420.

Vicente, K. J. (1999) Cognitive Work Analysis: Toward Safe, Productive, and Healthy ComputerBased Work, Lawrence Erlbaum Associates

Ylirisku, S. \& Buur, J. (2007) Designing with video: focusing the user-centred design process, Springer Ylirisku, S., Halttunen, V., Nuojua, J. \& Juustila, A. (2009) Framing design in the third paradigm. In Proceedings of the 27th international conference on Human factors in computing systems, 11311140, ACM

Zimmerman, J., Forlizzi, J. \& Evenson, S. (2007) Research through design as a method for interaction design research in $\mathrm{HCl}$. In Proceedings of the SIGCHI conference on Human factors in computing systems, San Jose, 493-502, ACM 